\title{
Psychobehavioral and Immunological Characteristics of HTLV-1 Carriers and Non-Carriers with Persistently Low Natural Killer Cell Activity
}

\author{
Akinori Masuda, Takami Matsuyama*, Mitchel Mitsuo Yokoyama**, \\ Shin-ichi NozoE*** and Chuwa TEI
}

\begin{abstract}
Objective To clarify the differences in immunological and psychobehavioral characteristics of HTLV-1 carriers and non-carriers with persistently low natural killer (NK) cell activity.

Methods The individuals with persistently low NK cell activity were divided into HTLV-1 carriers and non-carriers. NK cell activity, lymphocytic proliferation, lymphocyte subsets (CD4+, CD8+, CD16+, CD20+, CD56+), and psychobehavioral responses were examined.

Patients Of 296 outpatients with physical complaints, 30 patients with persistently low NK cell activity (10 HTLV1 carriers and 20 HTLV-1 non-carriers) and 20 healthy controls negative for HTLV-1 antibody and with normal NK cell activity were randomly selected.

Results In HTLV-1 carriers with persistently low NK cell activity, no significant differences were observed in NK cell subsets (CD16+ and CD56+) and psychobehavioral responses compared with the healthy controls. In HTLV-1 non-carriers, NK cell subsets were significantly low, and depression, anxiety and fatigue were significantly greater than in healthy controls.

Conclusions These findings suggest that persistently low NK cell activity in HTLV-1 carriers might be reduced due to the HTLV-1 infection. On the other hand, the reduction in the NK cell activity in HTLV-1 non-carriers appears to be related to depression, anxiety, and fatigue.

(Internal Medicine 39: 885-890, 2000)
\end{abstract}

Key words: NK cell subsets, psychobehavioral responses, HTLV-1 infection

\section{Introduction}

Natural killer (NK) cells are referred to as large granular lymphocytes (LGL) expressing CD16 (Fc $\gamma \mathrm{R}$ III) and CD56 (Leu19/NKH-1) as surface markers. The NK cell functions include immunological surveillance against tumors, control of viral infections, production of cytokines, and regulation of killer activity. Impaired NK cell activity can generally be identified in patients with congenital immunodeficiency $(1,2)$, malignant tumors (3), autoimmune diseases (4), acquired immunodeficiency syndrome (AIDS) $(5,6)$, HTLV-1-associated myelopathy/tropical spastic paraparesis (HAM/TSP) (7), chronic fatigue syndrome (CFS) $(8)$, depression $(9,10)$, and by stress (11). In addition to these conditions, the NK cell activity is known to be reduced in healthy individuals who exhibit a high Hassles frequency and a high confusion score (12). We have previously reported a significant decrease in NK cell activity in adults with chronic fatigue (13). Such evidence that latent immunological depression is present (14) and that the NK cell activity is reduced $(15,16)$ in carriers of human T-cell leukemia virus type 1 (HTLV-1) has been reported by several investigators. However, other cellular immune functions, viral antibody levels, or psychobehavioral responses of HTLV-1 carriers with persistently low NK cell activity have not yet been discussed.

Since Kagoshima, in the southwestern part of Japan is noted as a high cluster (about 8\%) of HTLV-1 carriers (17), the frequency of the carriers among individuals with low NK cell activity was noted. The purpose of this study was to clarify the differences in immunological and psychobehavioral characeristics of HTLV-1 carriers and non-carriers with persistently low NK cell activity.

\footnotetext{
From the First Department of Internal Medicine, *the Department of Medical Zoology, ${ }^{* * *}$ the Department of Psychosomatic Medicine, Kagoshima University, Kagoshima and **Kurume University School of Medicine, Kurume

Received for publication February 4, 2000; Accepted for publication June 4, 2000

Reprint requests should be addressed to Dr. Akinori Masuda, the First Department of Internal Medicine, Kagoshima University, 8-35-1, Sakuragaoka, Kagoshima 890-0075
} 


\section{Methods}

\section{Subjects}

The NK cell activity of 296 outpatients were examined. These patients consulted the internal medicine or psychosomatic medicine due to general fatigue, dizziness, headache, nausea, palpitation, difficulty in breathing, chest pain, abdominal pain, diarrhea, or low grade fever and underwent detailed examination, but no organic disease was detected. Twenty-eight patients had been treated at a previous hospital with drugs for controlling gastric or intestinal function, Chinese medicine, or minor tranquilizer under a diagnosis of gastritis, irritable colon syndrome, autonomic imbalance or climacteric disturbance. The NK cell activity was analyzed by using an effector/target cell ratio of $20: 1$ and $10: 1$ in 70 healthy adults, setting normal values (mean $\pm \mathrm{SD}$ ). The outpatients whose values were less than the mean-SD on two measurements at a three-month interval were selected as the persistently low NK cell activity group. This group was composed of patients who were positive for anti-HTLV-1 antibody (HTLV-1 carriers) and those who were negative (non-carriers). A healthy control group $(n=20)$ with NK cell activity values within mean \pm SD and without anti-HTLV-1 antibody was selected.

\section{Procedure}

Anti-HTLV-1 antibody was screened using the particle agglutination (PA) test, and HTLV-1 provirus DNA incorporation was examined by Southern blot analysis. Those who tested positive in the PA test without HTLV-1 provirus DNA incorporation were regarded as HTLV-1 carriers.

The number of leukocytes and lymphocytes in the peripheral blood was determined. The cellular immune functions were examined by determination of NK cell activity, lymphocytes subsets (CD3+, total T-cell; CD4+, helper/inducer T-cell; CD8+, suppressor/cytotoxic T-cell; CD16+, NK cell; CD20+, B-cell; CD56+; NK cell) and lymphocyte proliferation. NK cell activity was measured using the ${ }^{51} \mathrm{Cr}$-release assay using K-562 cells as target. The effector cells: target cell ratios were set at $10: 1$ and $20: 1$. The NK cell activity of the three control subjects was measured on every occasion for interassay control. Lymphocyte subsets were analyzed using a FACScan (Becton Dickinson, San Jose, CA) and the gates were set for lymphocytes. Lymphocyte proliferation test was performed with or without the stimulation with phytohemagglutinin (PHA), concanavalin $\mathrm{A}$ (Con A), or pokeweed mitogens (PWM).

The measurement of the antibodies to Epstein-Barr virus (EBV), early antigen (EA), viral capsid antigen (VCA), and nuclear antigen (NA) was performed with indirect immunofluorescence. The antibodies to herpes simplex virus (HSV) and cytomegalovirus (CMV) were assayed by complement fixation tests. All blood samples were obtained between ten and twelve AM to adjust for circadian periodicity.

We used the Japanese versions of Cornell Medical Index (CMI) $(18,19)$ to analyze psychophysical subjective symptoms. The psychobehavioral response was evaluated with the
Maudsley Personality Inventory (MPI) test (20) while depression was assessed using the Zung Self-Rating Depression Scale (SDS) (21). The Japanese translated versions of these tests are routinely used throughout Japan.

\section{Data analyses}

The results were expressed as mean $\pm \mathrm{SD}$. Data analysis among the three groups was performed with one-way analysis of variance, and $p$ values of $<0.05$ were considered statistically significant. When significant differences were indicated, Bonferroni's test was used to test for the mean differences among the three groups.

\section{Results}

\section{The prevalence of outpatients with persistently low NK cell activity and HTLV-1 carriers}

The NK cell activity was persistently a low level in 30 of the 296 outpatients examined. The prevalence of asymptomatic HTLV-1 carriers was 20 (7\%) of the 296 outpatients. The NK cell activity of HTLV-1 carriers $(n=20)$ was a low level compared with healthy controls $(\mathrm{p}<0.05)$ as previously reported $(15,16)$. Surprisingly, $10(33.3 \%)$ of 30 persistently low NK cell activity outpatients were asymptomatic HTLV-1 carriers.

We divided the 30 patients who showed persistently low NK cell activity into HTLV-1 carriers $(n=10)$ and non-carriers $(n=20)$. None of these subjects had been receiving medications and no significant differences in the age nor sex among the three groups could be identified (Table 1).

\section{Psychobehavioral responses of persistently low NK cell ac- tivity group and healthy controls}

The NK cell activity has been known to be reduced by depression, stress, and fatigue $(9,11,13)$. Therefore, we examined the psychobehavioral responses between the HTLV-1 carriers and non-carriers.

Based on the CMI test, the HTLV-1 non-carriers of persistently low NK cell activity group presented with physical complaints more frequently than those of the other two groups $(\mathrm{F}=3.5, \mathrm{p}=0.04)$, especially in marked fatigue $(\mathrm{F}=9.9, \mathrm{p}=0.0003)$ and a high frequency of diseases $(\mathrm{F}=7.9, \mathrm{p}=0.001)$ (Table 2). Furthermore, the same group exhibited more psychological complaints $(\mathrm{F}=5.5, \mathrm{p}=0.006)$ such as maladjustment $(\mathrm{F}=9.7$, $\mathrm{p}=0.0004)$, depression $(\mathrm{F}=7.0, \mathrm{p}=0.006)$, anxiety $(\mathrm{F}=3.1$, $\mathrm{p}=0.05)$, and tension $(\mathrm{F}=4.0, \mathrm{p}=0.03)$ which were significantly severe compared with the healthy controls. The HTLV-1 noncarriers showed significantly higher SDS than the other two groups $(\mathrm{F}=4.2, \mathrm{p}<0.05)$. In the MPI test, the HTLV-1 non-carriers showed significantly high scores in neuroticism compared with the other two groups $(\mathrm{F}=3.6, \mathrm{p}=0.04)$, whereas no significant differences were observed in extraversion among the three groups.

No significant differences were observed between the HTLV1 carriers with persistently low NK cell activity group and healthy controls in the use of parameters of the CMI test. 
Table 1. Age, Sex, and NK Cell Actitity of Persistently Low NK Cell Activity Group and Healthy Controls

\begin{tabular}{lccccc}
\hline & & \multicolumn{2}{c}{ LNKG $^{1)}$} & & \\
\cline { 3 - 4 } & $\begin{array}{c}\text { Control } \\
(\mathrm{n}=20)\end{array}$ & $\begin{array}{c}\text { HTLV-1 }(-) \\
(\mathrm{n}=20)\end{array}$ & $\begin{array}{c}\text { HTLV-1 }(+) \\
(\mathrm{n}=10)\end{array}$ & $\mathrm{F}$ & $\mathrm{P}$ \\
\hline Age & $42.1 \pm 8.1$ & $40.2 \pm 12.7$ & $49.0 \pm 11.3$ & 2.30 & $\mathrm{~ns}$ \\
Male/Female & $10 / 10$ & $7 / 13$ & $4 / 6$ & - & $\mathrm{ns}$ \\
NK activity $(\%)$ & & & & & \\
E/T=10:1 & $25.9 \pm 11.7$ & $4.2 \pm 2.5$ & $5.0 \pm 3.3$ & 46.2 & $<0.0001$ \\
\multicolumn{1}{c}{$20: 1$} & $35.7 \pm 13.9$ & $5.7 \pm 2.8$ & $7.2 \pm 3.1$ & 62.8 & $<0.0001$ \\
\hline
\end{tabular}

$\mathrm{LNKG}^{1)}$ : persistently Low NK cell activity group. HTLV-1 (-): HTLV-1 non-carriers. HTLV-1 (+): HTLV-1 carriers.

Table 2. Psychobehavioral Responses of Persistently Low NK Cell Activity Group and Healthy Controls

\begin{tabular}{lccccc}
\hline & & \multicolumn{2}{c}{ LNKG } & \\
\cline { 3 - 4 } & & & & \\
\cline { 3 - 4 } & Control & HTLV-1 (-) & HTLV-1 (+) & & \\
\hline CMI & & & & & \\
$\quad$ Physical complaint & $23.7 \pm 12.6$ & $39.3 \pm 23.1^{*}$ & $25.4 \pm 16.9$ & 3.5 & 0.04 \\
Fatigue & $1.1 \pm 1.2$ & $3.5 \pm 2.1^{* * *}$ & $1.4 \pm 1.5$ & 9.9 & 0.0003 \\
Mental complaint & $4.8 \pm 5.6$ & $14.8 \pm 10.9^{* *}$ & $13.0 \pm 11.2$ & 5.5 & 0.006 \\
Maladjustment & $0.8 \pm 1.7$ & $4.5 \pm 3.4^{* * *}$ & $1.6 \pm 2.0$ & 9.7 & 0.0004 \\
Depression & $0.1 \pm 0.2$ & $1.7 \pm 2.2^{* *}$ & $0 \pm 0$ & 7.0 & 0.003 \\
Anxiety & $0.7 \pm 1.2$ & $2.1 \pm 1.5^{*}$ & $1.9 \pm 3.0$ & 3.1 & 0.05 \\
Irritability & $0.9 \pm 1.1$ & $2.1 \pm 1.7$ & $2.1+2.1$ & 3.1 & 0.05 \\
Anger & $1.6 \pm 2.5$ & $2.6 \pm 2.9$ & $2.4+3.2$ & 0.6 & $\mathrm{~ns}$ \\
$\quad$ Tension & $0.3 \pm 0.6$ & $1.9 \pm 2.3^{*}$ & $1.0 \pm 1.5$ & 4.0 & 0.03 \\
$M P I$ & & & & & \\
Extraversion & $30.2 \pm 13.2$ & $25.5 \pm 14.3$ & $29.3 \pm 4.9$ & 0.5 & $\mathrm{~ns}$ \\
$\quad$ Neuroticism & $14.0 \pm 8.5$ & $24.3 \pm 12.7^{*}$ & $15.9 \pm 5.0$ & 3.6 & 0.04 \\
$S D S$ & $39.6 \pm 5.8$ & $48.5 \pm 12.4^{*}$ & $39.3 \pm 7.0$ & 4.2 & 0.03 \\
\hline
\end{tabular}

${ }^{*} \mathrm{p}<0.05, * * \mathrm{p}<0.01,{ }^{* * *} \mathrm{p}<0.001$ compared with control.

Lymphocyte proliferative responses and lymphocyte subsets in peripheral blood of persistently low NK cell activity group and healthy controls

No significant differences were observed in the peripheral blood leukocyte and lymphocyte counts among the three groups. Likewise, there were no significant differences in lymphocyte proliferation induced by PHA, ConA, and PWM among the three groups. However, spontaneous lymphocyte proliferation was significantly increased in the HTLV-1 carriers in the persistently low NK cell activity group, consistent with a previous reports $(22,23)$ (Table 3 ).

Regarding the lymphocyte subsets, the proportions of CD3+, CD4+, CD8+, and CD20+ cells showed no distinction among the three groups (Table 4). However, the proportions of CD16+ and CD56+ cells, characterizing NK cell subsets, were significantly lower in the HTLV-1 non-carriers than in the other two groups $(\mathrm{F}=8.37, \mathrm{p}=0.0008$ and $\mathrm{F}=8.0, \mathrm{p}=0.001)$. No significant differences, however, in the proportions of CD16+ and CD56+ cells between the HTLV-1 carriers and the healthy controls were found.

\section{Viral antibody titers of persistently low NK cell activity group and healthy controls}

The geometric mean titers of VCA-IgG were higher in persistently low NK cell activity group than in the healthy controls ( $\mathrm{p}=0.03)$. The CMV-IgG level was also higher in the persistently low NK cell activity group than in the healthy controls $(p=0.02)$, although no significant differences were observed between the HTLV-1 carriers and non-carriers. The HSVIgG antibody level was not significantly different among the three groups (Table 5). 
Table 3. Lymphocyte Proliferative Responses in Peripheral Blood of Persistently Low NK Cell Activity Group and Healthy Controls

\begin{tabular}{lcccccc}
\hline & & \multicolumn{2}{c}{ LNKG } & & \\
\cline { 3 - 4 } & Control & HTLV-1 (-) & HTLV-1 (+) & & P \\
\hline PHA & $103,229 \pm 30,172$ & $103,835 \pm 30,993$ & $95,758 \pm 28,959$ & 0.2 & ns \\
ConA & $71,291 \pm 25,882$ & $60,680 \pm 23,312$ & $58,420 \pm 21,614$ & 1.3 & ns \\
PWM & $21,770 \pm 12,020$ & $15,808 \pm 6,365$ & $16,094 \pm 4,952$ & 2.5 & ns \\
Control $^{2)}$ & $954 \pm 338$ & $643 \pm 197$ & $2,667 \pm 1,344 * * * *$ & 34.2 & $<0.0001$ \\
\hline
\end{tabular}

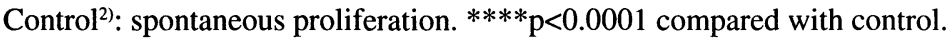

Table 4. Proportions of Lymphocyte Subsets in Peripheral Blood of Persistently Low NK Cell Activity Group and Healthy Controls

\begin{tabular}{lccccc}
\hline & & \multicolumn{2}{c}{ LNKG } & & \\
\cline { 3 - 4 }$\%$ & Control & HTLV-1 (-) & HTLV-1 (+) & F & P \\
\hline CD3+ & $66.4 \pm 11.2$ & $68.3 \pm 8.3$ & $70.3 \pm 11.8$ & 1.9 & ns \\
CD4+ & $40.0 \pm 10.4$ & $44.8 \pm 7.8$ & $45.9 \pm 7.6$ & 2.1 & ns \\
CD8+ & $28.6 \pm 7.7$ & $28.8 \pm 7.6$ & $26.1 \pm 5.4$ & 0.5 & ns \\
CD4/CD8 & $1.6 \pm 0.9$ & $1.7 \pm 0.7$ & $1.8 \pm 0.6$ & 0.4 & $n s$ \\
CD20+ & $11.9 \pm 4.7$ & $10.9 \pm 3.2$ & $12.5 \pm 4.6$ & 0.5 & ns \\
CD16+ & $15.3 \pm 7.9$ & $6.8 \pm 2.9 * * *$ & $11.7 \pm 7.7$ & 8.4 & 0.0008 \\
CD56+ & $22.5 \pm 9.5$ & $11.7 \pm 4.9 * * *$ & $18.2 \pm 8.6$ & 8.0 & 0.001 \\
\hline
\end{tabular}

$* * * \mathrm{p}<0.001$ compared with control.

Table 5. Virus Antibody Titers of Persistently Low NK Cell Activity Groups and Healthy Controls

\begin{tabular}{|c|c|c|c|c|c|}
\hline & \multirow[b]{2}{*}{ Control } & \multicolumn{2}{|c|}{ LNKG } & \multirow{2}{*}{$\mathrm{F}$} & \multirow{2}{*}{$P$} \\
\hline & & HTLV-1 (-) & HTLV-1 (+) & & \\
\hline EB VCA-IgG & $137 \pm 90$ & $197 \pm 99 *$ & $224 \pm 88^{*}$ & 4.3 & 0.03 \\
\hline HSV-IgG & $14 \pm 12$ & $17 \pm 13$ & $23 \pm 23$ & 0.9 & ns \\
\hline CMV-IgG & $20 \pm 12$ & $38 \pm 24 *$ & $35 \pm 18 *$ & 4.6 & 0.02 \\
\hline
\end{tabular}

${ }^{*} \mathrm{p}<0.05$ compared with control.

\section{Discussion}

The percentage of HTLV-1 carriers in their 40's living in Kagoshima prefecture is reported to be about $10 \%$ (24). In this study, the percentage of HTLV-1 carriers in outpatients was $7 \%$. We found a higher percentage (33\%) of HTLV-1 carriers in the persistently low NK cell activity group.

Yu et al (25) reported that low NK cell activity and the decrease in NK cell subsets (CD16+, CD56+, CD16-CD56+) were found in asymptomatic HTLV-1 carriers. They concluded that the decrease in NK cell activity might have been related to the decreased cell numbers and not to the NK cell function itself. Our results, however, indicated that the NK cell subsets (CD16+, CD56+) were not reduced in HTLV-1 carriers with persistently low NK cell activity compared with the healthy controls. The differences between the results of Yu et al (25) and the present results might be due to the selection of the subjects. Our subjects were asymptomatic HTLV-1 carriers with persistently low NK cell activity, whereas the subjects of the study of Yu et al were asymptomatic HTLV-1 carriers.

No significant changes were observed in the HTLV-1 carriers with persistently low NK cell activity in psychobehavioral responses that affect the NK cell activity such as depression, anxiety, and fatigue compared with the healthy controls. Furthermore, the NK cell subsets were not reduced in this group compared with the healthy controls. These findings indicate that HTLV-1 may exert a certain impact upon NK cell activity. Since HTLV-1 has been demonstrated to infect NK cells activated in vitro by anti-CD16 mAb (26), the present results suggest that in HTLV-1 carriers with persistently low NK cell activity, the HTLV-1 virus might infect NK cells and impair NK cell function without reducing the number of NK cells. The persistent decrease in NK cell activity in about $50 \%$ of the HTLV-1 carriers also suggests impairment of NK cell function by HTLV-1 virus.

Similarly, a reduction in the activity but not in the number 
of NK cells has already been reported in patients with another retrovirus-related entity, aquired immunodeficiency syndrome (AIDS) (27). In this syndrome, the decrease of NK cell activity is ascribed hypothetically to HIV infection of the NK cells (28). The mechanism of HIV infection was speculated to be through the infection of NK cells by human herpes virus 6 (HHV-6) which induces expression of CD4 markers, thus promoting HIV infection (29).

The EB virus-related antibody levels are used as indices for the estimation of changes in immune function, and VCA-IgG is reported to increase when abnormalities are present in NK cells (30). The EB VCA-IgG and CMV-IgG were higher in both HTLV-1 carriers and non-carriers of persistently low NK cell activity group than in the healthy controls. This result suggests that the high antibody titers against these viruses is due to the persistently low NK cell activity and not due to the HTLV1 infection.

In HTLV-1 non-carriers with persistently low NK cell activity, NK cell subsets (CD16+, CD56+) were reduced. As for psychobehavioral responses, those belonging to this group complained of many physical and mental symptoms. Particularly, fatigue was frequently noted in various complaints. Anxiety, depression, tension, and maladjustment were characteristic in the mental complaints and neuroticism was noted in this group. From these findings, the NK cell activity is considered to be reduced with a decrease in the number of NK cells due to the psychobehavioral characteristics in HTLV-1 non-carriers with persistently low NK cell activity.

Interleukin-1 (IL-1), which transmits immunological information to the central nervous system, is produced under stress (31). IL-1 also directly promotes secretion of corticotropinreleasing-hormone (CRH) from the hypothalamus, leading to the activation of the hypothalamo-pituitary-adrenal (HPA) axis (32). Cortisol, produced by the activated HPA axis, was reported to inhibit the NK cell activity (33). CRH activates the sympathetic system, and increased norepinephrine suppresses the NK cell activity (34). The hypothalamus is known to play a role of physiological pacemaker by regulating the NK cell activity via neuroendocrine hormones (35). CRH appears to be produced in excess under stimulation by IL-1, if stress is not managed appropriately and chronic fatigue or depression and anxiety persist. From the neuroimmunological viewpoint, it is thought that $\mathrm{CRH}$ causes reduction in the number and the activity of NK cells.

NK cells are known to have antiviral activities and to play a role in the pathogenesis of viral infections. These effector functions allow the possibility that NK cells may contribute to the long latent period characteristics of HTLV-1 infection and the onset of HTLV-1 associated diseases, although the role of NK cells in the pathogenesis of HTLV-1 infection remains to be elucidated. From this study, the function and the number of NK cells are affected by mental responses such as depression, anxiety and physical conditions such as fatigue as well as HTLV-1 infection. Since HTLV-1 may infect NK cells and impair NK cell function, further evaluation of these processes including follow-up of HTLV-1 carriers is anticipated.
Acknowledgements: The authors thank Hideo Otubo, MD, for technical assistance and Naomichi Arima, MD, for preparation of the manuscript.

\section{References}

1) Roder JC, Haliotis T, Klein M, et al. A new immunodeficiency disorder in humans involving NK cells. Nature 284: 553-555, 1980.

2) Sullivan JL, Byron KS, Brewster FE, Purtilo DT. Deficient natural killer cell activity in X-linked lymphoproliferative syndrome. Science 210: 543$545,1980$.

3) Steinhauer EH, Doyle AT, Reed J, Kadish AS. Defective natural cytotoxicity in patients with cancer: Normal number of effector cells but decreased recycling in patients with advanced disease. J Immunol 129: 22552259, 1982.

4) Oshimi K, Gonda N, Sumiya M, Kano S. Effects of corticosteroid on natural killer cell activity in systemic lupus erythematosus. Clin Exp Immunol 40: 83-88, 1980.

5) Rook AH, Masur H, Lane HC, et al. IL-2 enhances the depressed natural killer and cytomegalovirus-specific cytotoxic activities of lymphocytes from patients with the acquired immune deficiency syndrome. J Clin Invest 72: 398-403, 1983.

6) Katz JD, Mitsuyasu R, Gottlieb MS, Lebow LT, Bonavida B. Mechanism of defective NK cell activity in patients with aquired immunodeficiency syndrome (AIDS) and AIDS-related complex. J Immunol 139: 55-60, 1987.

7) Fujihara K, Itoyama Y, Yu F, Kubo C, Goto I. Cellular immune surveillance against HTLV-1 infected T lymphocytes in HTLV-1 associated myelopathy/tropical spastic paraparesis (HAM/TSP). J Neurol Sci 105: 99-107, 1991.

8) Gupta S, Vayuvegula B. A comprehensive immunological analysis in chronic fatigue syndrome. Scand J Immunol 33: 319-327, 1991.

9) Irwin M, Daniels M, Bloom ET, Smith TL, Weiner H. Life events, depressive symptoms, and immune function. Am J Psychiatry 144: 437$441,1987$.

10) Irwin M, Smith TL, Gillin JC. Low natural killer cytotoxicity in major depression. Life Sci 41: 2127-2133, 1987.

11) Locke SE, Kraus L, Leserman J, Hurst MW, Heisel JS, Williams RM. Life change stress, psychiatric symptoms, and natural killer cell activity. Psychosom Med 46: 441-453, 1984.

12) Levy SM, Herberman RB, Simons A, et al. Persistently low natural killer cell activity in normal adults: immunological, hormonal and mood correlates. Nat Immun Cell Growth Regul 8: 173-186, 1989.

13) Masuda A, Nozoe SI, Matsuyama T, Tanaka H. Psychobehavioral and immunological characteristics of adult people with chronic fatigue and patients with chronic fatigue syndrome. Psychosom Med 56: 512-518, 1994.

14) Nakada K, Kohakura M, Komoda H, Hinuma Y. High incidence of HTLV1 antibody in carriers of Strongyloides stercoralis. Lancet 1: 633, 1984 (letter).

15) Uno $H$, Kawano $K$, Matsuoka $H$, Tsuda $K$. Natural killer activity and antibody- dependent cellular cytotoxicity in patients with adult T-cell leukemia. Acta Haematologica Japonica 52: 730-739, 1989.

16) Tanaka $Y$, Oda $S$, Nagata $K$, et al. Immunological functions and phenotypes of peripheral blood lymphocytes from Human T-cell Leukemia Virus-1 carriers. J Clin Immunol 9: 477-484, 1989.

17) Yamaguchi K. Human T-lymphotropic virus type I in Japan. Lancet 343: 213-216, 1994.

18) Brodman K, Erdmann AJ, Lorge I, et al. The Cornell Medical Index-Health questionnaire 3. The evaluation of emotional disturbances. J Clin Psychol 8: 119-124, 1952.

19) Fukamachi $K$. The study on the Cornell Medical Index [1]. The characteristic of complaints of neurotic patients showed on the CMI. Fukuoka Igaku Zasshi 50: 2988-3000, 1959 (in Japanese with English summary).

20) Eysenck HJ. The questionnaire measurement of neuroticism and extraversion. Revista di Psicologia 50: 113-140, 1956.

21) Zung WW. A self-rating depression scale. Arch Gen Psychiatry 12: 63- 


\section{Masuda et al}

$70,1965$.

22) Kramer A, Jacobson S, Reuben JF, et al. Spontaneous lymphocyte proliferation in symptom-free HTLV-1 positive Jamaicans. Lancet 2: 923-924, 1989 (letter).

23) Itoyama $Y$, Minato $S$, Kira J, et al. Spontaneous proliferation of peripheral blood lymphocytes increased in patients with HTLV-1-associated myelopathy. Neurology 38: 1302-1307, 1988.

24) Maeda Y, Furukawa M, Takehara Y, et al. Prevalence of possible adult Tcell leukemia virus-carriers among volunteer blood donors in Japan: a nation-wide study. Int J Cancer 33: 717-720, 1984.

25) Yu F, Itoyama Y, Fujihara K, Goto I. Natural killer (NK) cells in HTLV-1associated myelopathy/tropical spastic paraparesis decrease in NK cell subset populations and activity in HTLV-1 seropositive individuals. J Nueroimmunol 33: 121-128, 1991.

26) Lo KM, Vivier E, Rochet N, et al. Infection of human natural killer (NK) cells with replication-defective human $\mathrm{T}$ cell leukemia virus type 1 provirus. Increased proliferative capacity and prolonged survival of functionally competent NK cells. J Immunol 149: 4101-4108, 1992.

27) Bonavida B, Katz J, Gottlieb M. Mechanism of defective NK cell activity in patients with acquired immunodeficiency syndrome (AIDS) and AIDSrelated complex. I. Defective trigger on NK cells for NKCF production by target cells, and partial restoration by IL 2 . J Immunol 137: 1157$1163,1986$.

28) Chehimi J, Bandyopadhyay S, Prakash K, et al. In vitro infection of natu- ral killer cells with different human immunodeficiency virus type 1 isolates. J Virol 65: 1812-1822, 1991.

29) Lusso P, Malnati MS, Garzino-Demo A, Crowley RW, Longo EO, Gallo RC. Infection of natural killer cells by human herpesvirus 6 . Nature 362 : 458-462, 1993.

30) Henle W, Henle G. Epstein-Barr virus -specific serology in immunologically compromised individuals. Cancer Res 41: 4222-4225, 1981.

31) Weiss JM, Sundar SK, Becker KJ, Cierpial MA. Behavioral and neural influences on cellular immune responses: Effects of stress and interleukin1. J Clin Psychiatry 50 Suppl: 43-53, 1989.

32) Tsagarakis S, Gillies G, Rees LH, Besser M, Grossman A. Interleukin-1 directly stimulates the release of corticotrophin releasing factor from rat hypothalamus. Neuroendocrinology 49: 98-101, 1989.

33) Holbrook NJ, Cox WI, Horner HC. Direct supression of natural killer cell activity in human peripheral blood leukocyte cultures by glucocorticoids and its modulation by interferon. Cancer Res 43: 4019-4025, 1983.

34) Irwin M, Hauger RL, Brown M, Britton KT. CRF activates autonomic nervous system and reduces natural killer cytotoxicity. Am J Physiol 255: R744-R747, 1988.

35) Mozzanica N, Finzi AF, Foppa S, Vignati G, Villa ML. Association between circadian rhythms of endogeneous hypothalamic opioid peptides and of natural killer cell activity. Int J Immunopharmacol 13: 317-321, 1991. 